\section{DENTAL UNIFORMS JUST FOR YOU}

Pastelli has been producing high quality uniforms for the dental profession since 1929.

One hundred percent made in Italy, the exclusive collections offer garments that are both stylish and comfortable, making them an ideal choice for a long day in surgery.

Available from RPA Dental, the various ranges cater for all of your individual needs and preferences, right down to cut, colour and size.

There are also a number of specialist fabrics to choose from. These include:

- Lino - second only to silk for quality and beauty

U Jersey - an extremely comfortable woven cotton fabric, which offers freedom of movement

- Polycotton - a cotton and polyester mix that is both strong and easy to maintain

- Pet wash and wear - a non-iron breathable and ecological material.

With many more textiles available to pick from, you are sure to find what you are looking for with Pastelli.

To find out more about the different collections and styles available, contact RPA Dental today. To see how RPA Dental can transform your dental practice, call 08000933975 , visit the website www. dental-equipment.co.uk or email jo@rpadental.net.
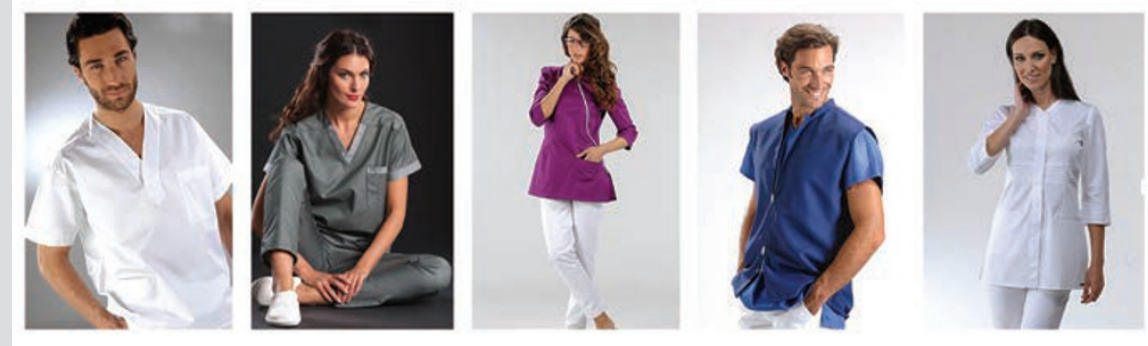

\title{
ARE YOU SITTING COMFORTABLY?
}

Most of us, without even realising it, are damaging our spines by the way we sit at work. For dental professionals, this is a serious issue, since musculoskeletal disorders are the main reasons for early retirement in the profession.

In other words, simply sitting down could be affecting the quality of your work and cutting years off your healthy career.

A-dec has a solution. Its range of ergonomic stools, including the premier A-dec 500 range, have been specially designed to provide dental professionals with an effective and comfortable seating option. A dynamic system, the A-dec 500 stool has three layers of flexible material designed to conform to your every shift and movement, providing you with unprecedented support and comfort.

What's more, the stool features for points of adjustability, enabling you to find the correct posture - and precise increments of movement can be made easily throughout the day to ensure you are sitting comfortably no matter what.

The A-dec 500 stool also features a variety of innovative optional features that increase its ergonomic efficacy. From additional foot pedestals to encourage the healthier 'athletic' stance to swingout armrests for optimum support, the stool has everything you need to practise dentistry safely and comfortably.

Visit www.a-dec.co.uk or call 0800233285.
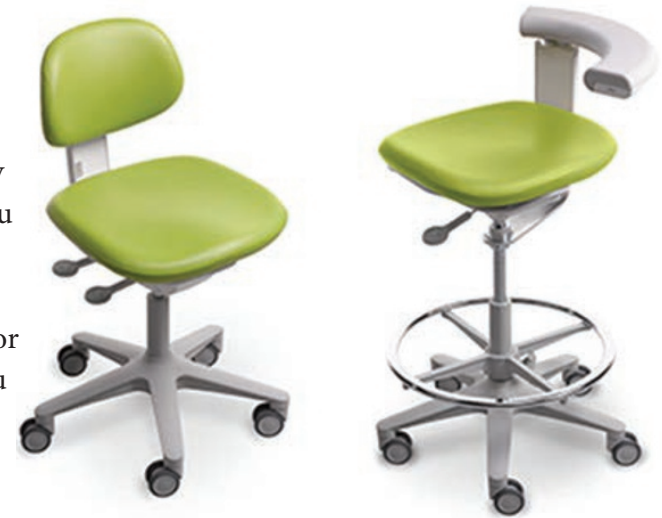

BUILD A BRIGHTER SURGERY

Could your practice do with a little bit of colour? Surgery Express has just the thing. The Vibrenté range of colourful dental i consumables, available exclusively I through Surgery Express, has a wide , choice of different essential items in a variety of bright and bold colours. Products include: nitrile gloves, disposable bibs, squat cups, paper tray liners, disposable aspirator tips, saliva ejectors and face masks.

Whatever the colour scheme of your surgery, the Vibrenté range will have something that matches. All of its high quality items are durable and cost effective, making it even easier to add a little bit of personality and flair into your practice.

The benefits of colourful equipment are limitless. Not only will your practice be able to strengthen its brand and I aesthetic appeal, but the colours included I in the Vibrenté range have been proven to help enhance mood - meaning your patients will benefit too!

To make the most of Surgery I Express's first-rate service, order the Vibrenté range before $3 \mathrm{pm}$ and receive guaranteed next-day delivery.

For more information, visit www. surgery-express.co.uk, call on 08006888 992 or email sales@surgery-express.co.uk.

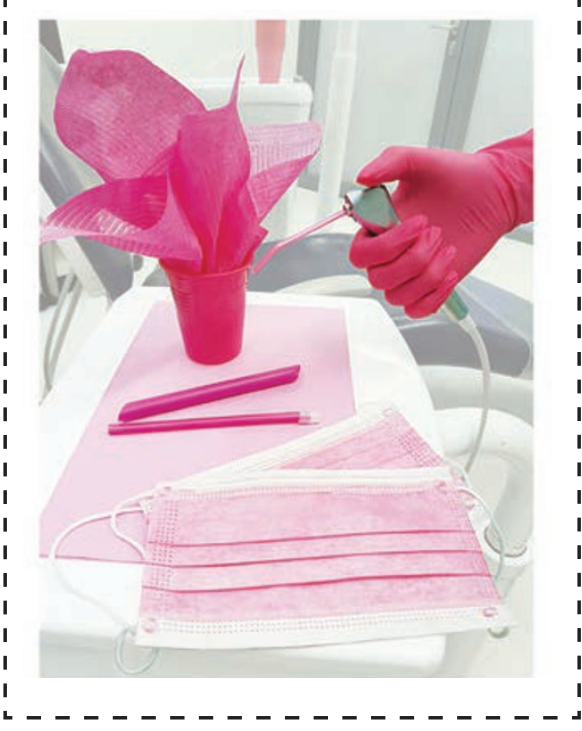
dental industry in BDJ Team, call Andy May on 02078434785 or emaila.may@nature.com. 